\title{
Intra-cluster globular clusters around NGC 1399 in Fornax? ${ }^{\star}$
}

\author{
Y. Schuberth ${ }^{1,2}$, T. Richtler ${ }^{1}$, L. Bassino ${ }^{3}$, and M. Hilker ${ }^{4}$ \\ 1 Universidad de Concepción, Departamento de Física, Casilla 160-C, Concepción, Chile \\ 2 Argelander-Institut für Astronomie, Universität Bonn, Auf dem Hügel 71, 53121 Bonn, Germany \\ e-mail: ylva@astro.uni-bonn.de \\ ${ }^{3}$ Facultad de Ciencias Astronómicas y Geofísicas, Universidad Nacional de La Plata, Paseo del Bosque S/N, 1900-La Plata, \\ Argentina and IALP-CONICET \\ 4 European Southern Observatory, Karl-Schwarzschild-Str. 2, 85748 Garching, Germany
}

Received 14 September 2007 / Accepted 16 October 2007

\section{ABSTRACT}

\begin{abstract}
Aims. We investigate whether the globular clusters (GCs) in the recently published sample of GCs in the Fornax cluster by Bergond and coworkers are indeed intra-cluster objects.

Methods. We combine their catalogue of radial velocity measurements with our CTIO MOSAIC photometry in the Washington system and analyse the relation of metal-poor and metal-rich GCs with their host galaxies.

Results. The metal-rich GCs appear to be kinematically associated with their respective host galaxies. The vast majority of the metal-poor GCs found in between the galaxies of the Fornax cluster have velocities that are consistent with their being members of the very extended NGC 1399 GC system. We find that when the sample is restricted to the most accurate velocity measurements, the GC velocity dispersion profile can be described with a mass model derived for the NGC 1399 GC system within $80 \mathrm{kpc}$. We identify one "vagrant" GC whose radial velocity suggests that it is not bound to any galaxy unless its orbit has a very large apogalactic distance.
\end{abstract}

Key words. galaxies: star clusters - galaxies: kinematics and dynamics - galaxies: elliptical and lenticular, cD galaxies: clusters: individual: Fornax cluster - galaxies: individual: NGC 1399

\section{Introduction}

The existence of intra-cluster globular clusters (ICGCs) - i.e. globular clusters (GCs) that are not bound to individual galaxies but, rather, move freely through the potential well of a galaxy cluster as a whole - was proposed by White (1987) and West et al. (1995). In the nearby $(D=19 \mathrm{Mpc})$ Fornax cluster of galaxies, ICGC candidates were identified as an excess population of GC candidates in the vicinity of dwarf galaxies (Bassino et al. 2003) and as a region of enhanced number density of GC candidates in between the central dominant galaxy NGC 1399 and its second-nearest (giant) neighbour, NGC 1387 (Bassino et al. 2006a, cf. their Fig. 9). Tamura et al. (2006) have performed a wide-field survey of the central Virgo cluster and detected an excess population of GC candidates far away from any major galaxy. The spectroscopic confirmation of these candidate ICGCs, however, is still pending.

Recently, Bergond et al. (2007) have presented the velocities of a sample of GCs in the Fornax cluster, with some objects having projected distances of more than $230 \mathrm{kpc}$ from NGC 1399. These authors labelled a significant fraction of their objects as ICGCs. One does have to bear in mind that, with the photometric study by Bassino et al. (2006a), it became clear that the very populous globular cluster system (GCS) of the central galaxy, NGC 1399, has an extent of at least $250 \mathrm{kpc}$, which is

* Table 2 is available in electronic form at the CDS via anonymous ftp to cdsarc.u-strasbg.fr $(130.79 .128 .5)$ or via http://cdsweb.u-strasbg.fr/cgi-bin/qcat?J/A+A/477/L9 comparable to the core radius of the cluster $\left(R_{\mathrm{King}}=0.7^{\circ} \simeq\right.$ 230 kpc, Ferguson 1989).

In this Letter, we present Washington photometry for 116 of the $149 \mathrm{GCs}$ of the Bergond et al. kinematic sample and demonstrate that the photometric and dynamical properties of the "ICGCs" are consistent with those GCs that belong to NGC 1399.

\section{The data set}

Below, we briefly describe the two data sets combined in this study. The table (Table 2) is available in electronic form.

\subsection{Kinematic data}

Bergond et al. (2007, hereafter B07+) used the FLAMES multiobject, fibre-fed spectrograph in the GIRAFFE/MEDUSA mode on the VLT to obtain medium-resolution spectra of 149 GCs in the central region of the Fornax cluster (see their Fig. 1 for the location of the objects). They determined the velocities via Fourier cross-correlation and assigned a quality flag to each measurement. "Class A" indicates a very secure measurement, while "Class B" measurements have larger uncertainties (see B07+ for details). These authors define as ICGCs those objects more than $1.5 d_{25}$ away from any bright Fornax galaxy ${ }^{1}$. In the following,

\footnotetext{
${ }^{1} d_{25}$ is the isophotal diameter of a galaxy at the level of $25 \mathrm{mag} / \operatorname{arcsec}^{2}$ in the $B$-band.
} 
Table 1. The combined data set: GC colours and environment.

\begin{tabular}{lrrrrrrrr}
\hline \hline & \multicolumn{2}{c}{ B07+ } & \multicolumn{2}{c}{ Red } & \multicolumn{2}{c}{ Blue } & \multicolumn{2}{c}{$N_{\text {Blue }} / N_{\text {Red }}$} \\
& \multicolumn{2}{c}{$(1)$} & \multicolumn{2}{c}{$(2)$} & \multicolumn{2}{c}{$(3)$} & \multicolumn{2}{c}{$(4)$} \\
\hline All & 149 & $(109)$ & 43 & $(35)$ & 73 & $(56)$ & 1.7 & $(1.6)$ \\
ICGC & 61 & $(45)$ & 11 & $(9)$ & 45 & $(36)$ & 4.1 & $(4.0)$ \\
$R_{\text {pro }} \leq 1.5 d_{25}$ & 88 & $(64)$ & 32 & $(26)$ & 28 & $(20)$ & 0.9 & $(0.8)$ \\
\hline
\end{tabular}

The first column gives the number of GCs with velocity measurements from the B07+ catalogue. The number of red and blue GCs is given in Cols. (2) and (3), respectively. In all columns, the number of "Class A" velocity measurements is given in parentheses.

we will use the term ICGC in this geometrical sense, unless otherwise stated.

\subsection{Photometric data}

As part of our programme studying the GCSs of ellipticals in the Fornax cluster, wide-field photometry in the metallicity-sensitive Washington system was obtained for several fields, using images from the CTIO MOSAIC camera with a field-of-view of $36^{\prime} \times$ $36^{\prime}$. The results have been presented in a series of papers (Dirsch et al. 2003; Bassino et al. 2006a,b) to which we refer the reader for details of the observations and data reduction. We then used these data to determine the magnitudes and $C-R$ colours for the GCs presented by $\mathrm{B} 07+$.

\subsection{The combined data set}

In total, 121 out of the 149 B07+ GCs (listed in their Table 1) were matched to objects in our photometry database (the remaining objects mostly lie in chip gaps of the undithered MOSAIC images or are too close to the galaxy centres). Of these $121 \mathrm{ob}-$ jects, all but five have colours that lie in the range $0.8 \leq C-R \leq$ 2.3, which was used for the GC candidate selection by Dirsch et al. (2003). Discarding the objects with deviant colours, we thus define a sample of $116 \mathrm{GCs}$ that have reliable photometry. Out of these, 56 are ICGCs according to the definition of B07+. The remaining 60 GCs are "masked objects", i.e. they are found within $1.5 d_{25}$ of a Fornax galaxy (see Fig. 1 of B07+).

The upper panel of Fig. 1 shows the colour-magnitude diagram for all B07+ objects with reliable Washington photometry together with the photometry of the NGC 1399 GCS presented by Dirsch et al. (2003). Following these authors, we adopt $C-R=1.55$ as the colour dividing red (metal-rich) from blue (metal-poor) GCs. Note that four ICGCs have extremely blue colours $(C-R \leq 1.0)$. The middle and bottom panels of Fig. 1 show the colour histograms for the "masked objects" and the ICGCs, respectively. One sees that the ICGCs are preferentially blue, while the GCs in the vicinity of the Fornax galaxies have a broad distribution of colours. Table 1 lists the number of red and blue GCs with reliable photometry found in the two different environments.

\section{Colours and kinematics of globular clusters}

Figure 2 compares the velocity distributions of the blue and red GCs. For comparison, the histogram of all 149 B07+ velocities is shown. The blue GCs (lower panel) show a clear peak near the velocity of NGC 1399. The velocity distribution of the red GCs (upper panel) peaks at a lower velocity. Note that of the five brightest galaxies in the survey area, besides NGC 1399, four have velocities that are lower than the NGC 1399 systemic

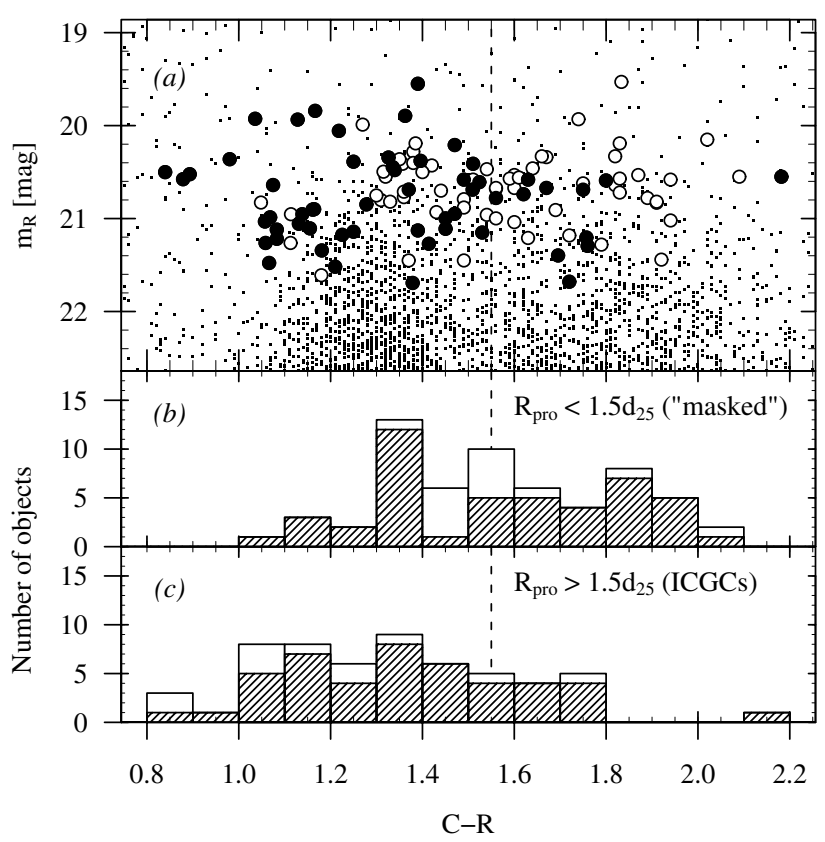

Fig. 1. MOSAIC photometry. The upper panel shows the colour magnitude diagram for the B07+ objects with MOSAIC photometry. Filled circles are ICGCs, and open circles represent GCs within $1.5 d_{25}$ of a giant Fornax member. The objects from the photometric catalogue of NGC 1399 GC candidates (central field) by Dirsch et al. (2003) are shown as dots. The middle and bottom panels show the colour histograms for the "masked" objects and the ICGCs, respectively. In both panels, the dashed histograms show the objects with "Class A" velocity measurements. The dashed line at $C-R=1.55$ shows the colour used to separate blue from red GCs (cf. Dirsch et al. 2003).

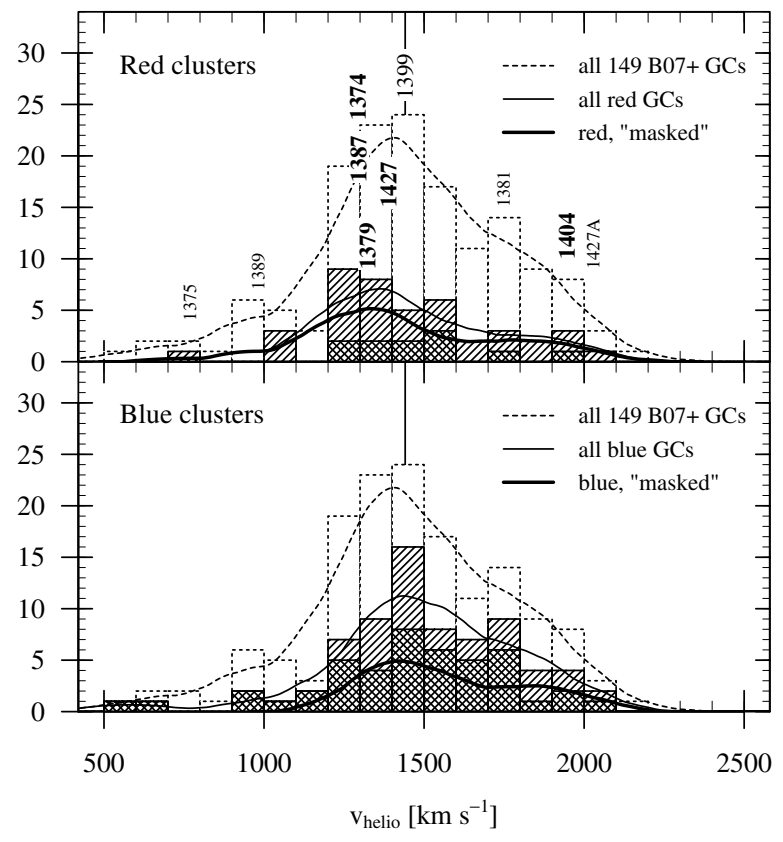

Fig. 2. Upper panel: velocities for all red GCs (dashed histogram) and red ICGCs (hashed). Lower panel: the same for the blue GCs. For reference, the distribution of all $149 \mathrm{GC}$ velocities from the B07+ catalogue is shown as a dotted histogram. The solid line is the systemic velocity of NGC 1399. The curves show Epanechnikov kernel density estimates for the samples indicated in the legend. In the upper panel, the systemic velocities of the 10 Fornax galaxies in the area covered by the B07+ study are shown by their corresponding NGC numbers. Boldface labels indicate the five brightest galaxies besides NGC 1399. 


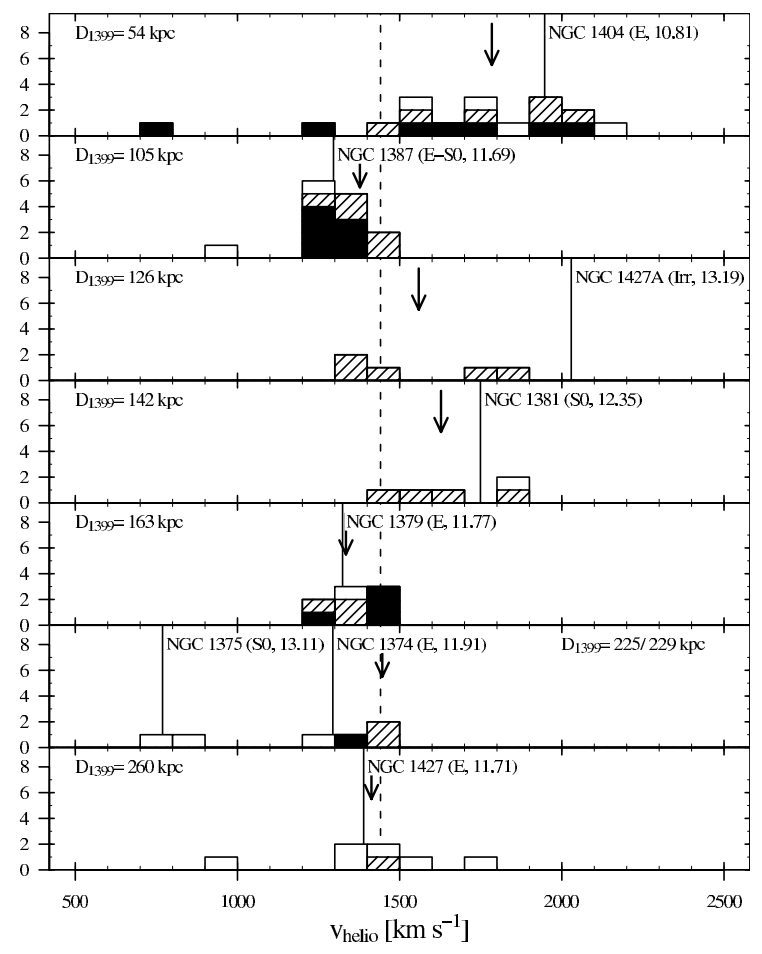

Fig. 3. Kinematics of the "masked" GCs. In each panel, the systemic velocity (solid lines) of the Fornax member is shown with the velocities of the objects within the corresponding $3 d_{25}$ diameter mask as applied by B07+. Solid and hashed bars represent red and blue GCs, respectively. Hollow histograms show the objects for which no MOSAIC photometry is available. Arrows indicate the mean velocity of the blue clusters in the panel. The galaxy type and the total apparent corrected $B$-magnitude as listed in the HyperLeda (Paturel et al. 2003) database are given in parentheses. In all panels, the systemic velocity of NGC 1399 is shown as a dashed line, and $D_{1399}$ denotes the projected distance from NGC 1399. For NGC 1389, no GCs were observed within its mask diameter.

velocity (1441 $\mathrm{km} \mathrm{s}^{-1}$ ), while only one (NGC 1404) has a higher velocity. Below, we study the kinematics of the GCs in the masked areas. The kinematics of the ICGCs are discussed in Sect. 3.2.

\subsection{The globular clusters in the vicinity of Fornax galaxies}

In total, 88 GCs are found in the masked areas centred on NGC 1399 and nine other Fornax galaxies. In Fig. 3 we show the velocity distributions of the latter (for NGC 1399, refer to Sect. 3.2). Each plot displays the velocities for the objects covered by the $3 d_{25}$-diameter mask of the corresponding Fornax member. The panels are sorted (from top to bottom) by projected distance from NGC 1399. In all panels, the systemic velocity of NGC 1399, and the velocity of the galaxy are shown. Moreover, the mean velocity of the blue GCs in a given panel is indicated.

With the exception of NGC 1404 whose GCS is projected onto that of NGC 1399 (and maybe even interacts with NGC 1399, e.g. Bekki et al. 2003 and references therein), the red GCs, if present, are always found within $100 \mathrm{~km} \mathrm{~s}^{-1}$ of the host galaxy. Furthermore, we note that all red GCs are associated with bright $\left(L_{B} \leq-19.5\right)$ early-type galaxies ${ }^{2}$.

${ }^{2}$ This is probably a consequence of the limiting magnitude of $V \simeq$ $22.2 \mathrm{mag}$. The spectroscopic survey only probes the brightest part of the GC luminosity function, and the fainter galaxies, hosting less populous GCSs, are simply less likely to possess a large number of bright GCs.
The velocities of the blue GCs, on the other hand, are not very strongly correlated with the systemic velocities of the galaxies. In fact, the velocity distributions of the blue GCs appear to be shifted towards the systemic velocity of NGC 1399, as can be seen from the arrows displayed in the panels. This effect is strongest for NGC 1381 and NGC 1427A, which both have high relative velocities with respect to NGC 1399. We suggest that the majority of the blue GCs observed in the vicinity of these two galaxies are associated with NGC 1399. For the masks covering NGC 1387, NGC 1379, and NGC 1427, it is not possible to make such a distinction since the velocities of these galaxies are within just $150 \mathrm{~km} \mathrm{~s}^{-1}$ of the NGC 1399 systemic velocity.

\subsection{NGC 1399 and the ICGCs}

The bottom panel of Fig. 4 shows the velocities of all objects labelled as ICGCs by B07+, together with the GCs within the $3 d_{25}$ area of NGC 1399, but excluding those within the mask centred on NGC 1404.

The majority of the ICGCs is blue, and only $20 \%$ of the ICGCs red (cf. Table 1). This is in accordance with the steeper slope of the number density profile found for the red GCs (Bassino et al. 2006a). One further notes that the red ICGCs ("Class A") have velocities within less than $280 \mathrm{~km} \mathrm{~s}^{-1}$ of the systemic velocity of NGC 1399. Their mean velocity is $1442 \pm$ $52 \mathrm{~km} \mathrm{~s}^{-1}$, and the dispersion is apparently very low. (For the 9 red "Class A" ICGCs, we find $\sigma_{\text {los }}=155 \pm 36 \mathrm{~km} \mathrm{~s}^{-1}$, but this value should be treated with caution, given the low number of data points.) The presence of red GCs at large distances from NGC 1399 is not unexpected after Bassino et al. found the red subpopulation of NGC 1399 GCs to extend to about 35'. The kinematics of the red ICGCs suggest that these objects are indeed part of the NGC 1399 GCS.

The blue ICGCs within $30^{\prime}$ ( $\simeq 165 \mathrm{kpc}$ ) of NGC 1399 are also clearly concentrated towards the systemic velocity of the central galaxy, albeit with a larger scatter, indicative of a higher velocity dispersion. For the 25 blue "Class A" ICGCs in this radial range, we find a mean velocity of $1474 \pm 53 \mathrm{~km} \mathrm{~s}^{-1}$ and a dispersion of $\sigma_{\mathrm{los}}=266 \pm 37 \mathrm{~km} \mathrm{~s}^{-1}$.

\section{Dynamics of the ICGCs}

Bergond et al. note that the velocity dispersion profile of the ICGCs exhibits a sharp rise beyond $\sim 150 \mathrm{kpc}$, almost reaching the value found for the dwarf galaxies in Fornax $\left(\sigma_{\text {dwarfs }}=\right.$ $429 \pm 41 \mathrm{~km} \mathrm{~s}^{-1}$, Drinkwater et al. 2001). In their view, this finding suggests a dynamic link between GCs and dwarf galaxies, supporting their interpretation as intra-cluster objects. It is interesting to review this statement in the context of the dynamics of the NGC 1399 GCS out to $80 \mathrm{kpc}$ (Richtler et al. 2004; Schuberth et al., in prep.).

In the upper panel of Fig. 4, we show two isotropic Jeans models derived from the kinematics of a sample of GCs within $80 \mathrm{kpc}$ (Richtler et al. 2007; Schuberth et al., in prep.), and a curve that corresponds to a halo that provides a good approximation to the mass profile of the Fornax cluster as given by Drinkwater et al. (2001). All models were calculated for the sum of the stellar mass of NGC 1399 and an NFW halo, i.e. $\varrho_{\mathrm{nfw}}(r)=\varrho_{\mathrm{s}}\left(\frac{r_{\mathrm{s}}}{r}\right)\left(1+\frac{r}{r_{\mathrm{s}}}\right)^{-2}$, with the parameters given in the legend. Furthermore, the upper panel of Fig. 4 shows the line-of-sight velocity dispersion profiles for two different samples, calculated for moving bins containing $20 \mathrm{GCs}$. The dashed line shows $\sigma_{\text {los }}(R)$ for all $87 \mathrm{GCs}$ in the lower panel (i.e. all 


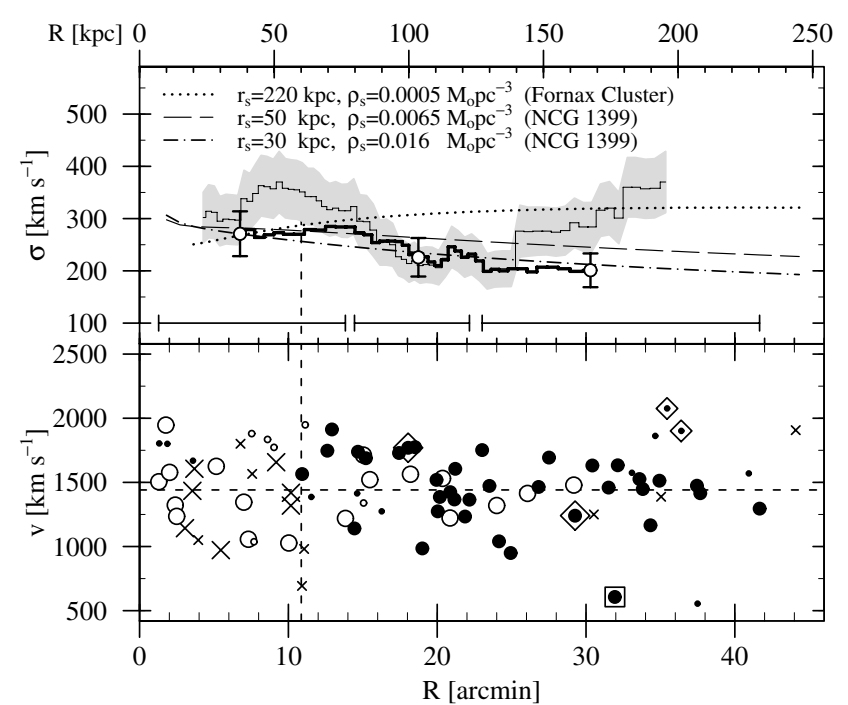

Fig. 4. Upper panel: line-of-sight velocity dispersion profiles. The thin solid line is the dispersion profile for all objects displayed in the lower panel, and the grey region shows the uncertainties. The thick solid line is the profile obtained when omitting all "Class B" objects and the most extreme "Class A" ICGC (indicated by a square in the lower panel). Both profiles were calculated using a moving bin containing 20 objects. The data points show the results for three independent bins, containing $20 \mathrm{GCs}$ each. The range of radial distances of the GCs used in a given bin are indicated by the horizontal bars. The dashed and dot-dashed curves are two models derived from the NGC 1399 GC dynamics within $80 \mathrm{kpc}$ (see text for details). The dotted curve corresponds to a halo which reproduces the mass profile of the Fornax cluster as derived by Drinkwater et al. (2001), (their Fig. 4, solid line). Lower panel: velocity vs. projected distance from NGC 1399 for 87 GCs (GCs within $1.5 d_{25}$ of other Fornax galaxies are not plotted). Large and small symbols are objects with "Class A" and "Class B" velocity measurements, B07+, respectively. Red and blue GCs are shown as open and filled circles, respectively. Crosses are objects for which no MOSAIC photometry is available. The four objects with unusually blue colours $(C-R \leq 1.0)$ are marked with diamonds. The square marks the "Class A" ICGC with the most extreme velocity. In both panels, the vertical dashed line shows the radius of the $3 d_{25}$-diameter mask $\left(1.5 d_{25}=10^{\prime} .9 \simeq 60 \mathrm{kpc}\right)$ of NGC 1399.

ICGCs and the GCs near NGC 1399 but excluding those within $1.5 d_{25}$ of NGC 1404). For $R \geq 10^{\prime}$, it corresponds to the profile shown in Fig. 3 of B07+. The second profile was calculated after discarding all "Class B" velocity measurements and the most extreme "Class A" velocity. This sample of "highquality" NGC 1399/ICGC velocities comprises 60 objects. The data points show this sample divided into three independent bins of $20 \mathrm{GCs}$.

Between 60 and $100 \mathrm{kpc}$, the dispersion profile of the "highquality" GCs has a much gentler slope than the one found when using all GCs, and it continues to decline, even where the full sample shows an increasing dispersion. We suggest that the sharp decrease in the velocity dispersion between 75 and $100 \mathrm{kpc}$ reported by B07+ is mainly due to one extremely low ("Class B") velocity at about $11^{\prime}$, which leads to large dispersion when included in the moving bin.

The sudden rise at $\sim 150 \mathrm{kpc}$, on the other hand, is caused by about six clusters, five of which have "Class B" velocity measurements. Two of these have surprisingly blue colours $(C-R=0.84$ and 0.88$)$, which are unusual for GCs. The deviant "Class A" object has a velocity of $607 \pm 9 \mathrm{~km} \mathrm{~s}^{-1}$. Such a large offset (of the order $800 \mathrm{~km} \mathrm{~s}^{-1}$, corresponding to four times the local velocity dispersion) from the cluster mean velocity is difficult to explain for a bound GC. Under the assumptions that this object is bound and that the observed radial velocity equals the pericentric velocity, we estimate the apogalactic distance (using the $r_{\mathrm{s}}=50 \mathrm{kpc}$ halo model of Fig. 4) to be of the order $r_{\text {apo }}>1 \mathrm{Mpc}$. For the more massive $\left(r_{\mathrm{s}}=220 \mathrm{kpc}\right)$ halo, which appears to be at variance with the data, one still obtains $r_{\text {apo }} \simeq 0.5 \mathrm{Mpc}$. From its photometry $\left(C-R=1.13, m_{R}=21.06\right)$, we derive, using $m-M=31.40$ and $V-R=0.5$, an absolute magnitude of $M_{V}=-9.87$. With the Harris \& Harris (2002) colour-metallicity relation, we obtain $[\mathrm{Fe} / \mathrm{H}]=-1.7$.

\section{Results and concluding remarks}

The red (metal-rich) GCs seem to be kinematically associated with their respective host galaxy or with NGC 1399. The population of ICGCs is predominantly blue, as expected from the shallower number density profile of the metal-poor NGC 1399 GCs. For the blue GCs, it is harder to determine whether a given GC "belongs" to a minor Fornax member or if it is part of the ICGC/NGC 1399 population. We conclude that the vast majority of the blue ICGCs in fact belong to the very extended NGC 1399 GCS. Out to at least $165 \mathrm{kpc}$, the velocity dispersion profile of the GCs is consistent with the mass profile derived from the dynamics of the NGC 1399 GCS within $80 \mathrm{kpc}$.

We propose to distinguish between intra-cluster GCs, i.e. GCs found at large distances from the galaxies in a cluster (i.e. a geometrical classification) and vagrant GCs, which are characterised by radial velocities suggesting that they are not bound to any galaxy in particular, but rather belong to the galaxy cluster as a whole. The example of Fornax illustrates the difficulties one faces when trying to identify vagrant clusters. The detection of stripped GCs is made difficult by the richness and extent of the NGC 1399 GCS and by its high velocity dispersion. For NGC 1404, which has a velocity of about $500 \mathrm{~km} \mathrm{~s}^{-1}$ with respect to NGC 1399, but a (projected) distance of only $\sim 50 \mathrm{kpc}$, it will be extremely hard to distinguish between a superposition along the line of sight and stripping. At about $100 \mathrm{kpc}$, NGC 1387 has a systemic velocity that is just $\sim 150 \mathrm{~km} \mathrm{~s}^{-1}$ lower than that of NGC 1399, making it hard to single out stripped GCs, although the photometry of Bassino et al. (2006a) suggests their presence. We suggest that a dynamical study of the NGC 1399/NGC 1404 and NGC 1387 region might yield evidence of tidal structures.

Acknowledgements. We thank the anonymous referee for useful comments. Y.S. acknowledges support from a German Science Foundation Grant (DFGProjekt HI-855/2). T.R. acknowledges support from the Chilean Center for Astrophysics, FONDAP No. 15010003. We acknowledge the use of the HyperLeda database (http://leda.univ-lyon $1 . \mathrm{fr}$ ).

\section{References}

Bassino, L. P., Cellone, S. A., Forte, J. C., \& Dirsch, B. 2003, A\&A, 399, 489 Bassino, L. P., Faifer, F. R., Forte, J. C., et al. 2006a, A\&A, 451, 789 Bassino, L. P., Richtler, T., \& Dirsch, B. 2006b, MNRAS, 367, 156 Bekki, K., Forbes, D. A., Beasley, M. A., \& Couch, W. J. 2003, MNRAS, 344, 1334

Bergond, G., Athanassoula, E., Leon, S., et al. 2007, A\&A, 464, L21

Dirsch, B., Richtler, T., Geisler, D., et al. 2003, AJ, 125, 1908

Drinkwater, M. J., Gregg, M. D., \& Colless, M. 2001, ApJ, 548, L139

Ferguson, H. C. 1989, AJ98, 367

Harris, W., \& Harris, G. 2002 AJ, 123, 3108

Paturel, G., Petit, C., Prugniel, P., et al. 2003 A\&A, 412, 45

Richtler, T., Dirsch, B., Gebhardt, K., et al. 2004, AJ, 127, 2094

Richtler, T., Schuberth, Y., Hilker, M., et al. 2007, A\&A, submitted

Tamura, N., Sharples, R. M., Arimoto, N., et al. 2006, MNRAS, 373, 601

West, M. J., Côté, P., Jones, C., et al. 1995, ApJ, 453, L77

White, R. E., III 1987, MNRAS, 227, 185 